\title{
Effects of syntactic category assignment on lexical ambiguity resolution in reading: An eye movement analysis
}

\author{
JOCELYN R. FOLK \\ Kent State University, Kent, Ohio \\ and \\ ROBIN K. MORRIS \\ University of South Carolina, Columbia, South Carolina
}

\begin{abstract}
Readers' eye movements were monitored as they read sentences containing lexically ambiguous words whose meanings share a single syntactic category (e.g., calf), lexically ambiguous words whose meanings belong to different syntactic categories (e.g., duck), or unambiguous control words. Information provided prior to the targetalways unambiguously specified the context-appropriate syntactic-category assignment for the target. Fixation times were longer on ambiguous words whose meanings share a single syntactic category than on controls, both when prior context was semantically consistent with the subordinate interpretation of a biased ambiguous word (Experiment 1) and when prior context was semantically neutral as to the intended interpretation of a balanced ambiguous word (Experiment 2). These ambiguity effects, which resulted from differences in difficulty with meaning resolution, were not found when the ambiguity crossed syntactic categories. These data indicate that, in the absence of syntactic ambiguity, syntactic-category information mediates the semantic-resolution process.
\end{abstract}

Ambiguity resolution is an important component of language comprehension. Readers and speakers routinely encounter ambiguity at the sentence level, when multiple interpretations of a sentence are possible, and at the lexical level, when the ambiguity resides in the interpretation of a single word. The majority of the research concerning lexical ambiguity has focused on the role of semantic context and meaning dominance in resolving ambiguity (Binder \& Morris, 1995; Dopkins, Morris, \& Rayner, 1992; Duffy, Morris, \& Rayner, 1988; Folk \& Morris, 1995; Rayner \& Duffy, 1986; Rayner \& Frazier, 1989; Rayner, Pacht, \& Duffy, 1994; Seidenberg, Tanenhaus, Leiman, \& Bienkowski, 1982; Sereno, Pacht, \& Rayner, 1992; Simpson \& Burgess, 1985; Simpson \& Foster, 1986; Simpson \& Kellas, 1988; Tabossi, 1988). In the present studies, we examined the effect of syntactic-category assignment on meaning resolution in structurally unambiguous contexts. To accomplish this, we directly compared the processing of lexically ambiguous words whose meanings share a single syntactic category (e.g., calf) with that of lexically ambiguous words when the word meanings are in different syntactic categories (e.g., duck). Our goal was to examine the influence of syntactic-category assignment on meaning resolution.

We thank Clanitra Stewart for her assistance on this project. Correspondence concerning this article should be addressed to J. R. Folk, Department of Psychology, P. O. Box 5190, Kent State University, Kent, OH 44242-0001 (e-mail: jfolk @ kent.edu).
Several researchers have suggested that semantic processing may be influenced by syntactic analysis. At one end of the spectrum, MacDonald, Pearlmutter, and Seidenberg (1994) proposed that lexical-and syntactic-ambiguity resolution engage common processing mechanisms. According to this view, the processor makes use of all available information to resolve ambiguities through constraint satisfaction. In contrast, O'Seaghdha $(1989,1997)$ proposed that syntactic-category assignment precedes semantic analysis so that, when syntactic-category assignment fails, further semantic processing is delayed (see also Gorrell, 1989). This view, based largely on the finding that syntactic context effects are independent of semantic relatedness and are impervious to semantic anomaly, implies a level of separation between syntactic and semantic knowledge. Finally, Boland (1993) espoused a hybrid view that syntactic and semantic analyses are distinct processes, but that under some circumstances semantic processing may be helped by unambiguous syntactic information.

In the present studies, we investigated the relationship between syntactic-category assignment and meaning resolution processes by directly comparing lexically ambiguous words whose meanings share a single syntactic category with lexically ambiguous words whose meanings belong to different syntactic categories. This afforded us the opportunity to examine meaning resolution processes under circumstances in which syntactic information could influence semantic resolution (i.e., for lexical ambiguity that crosses syntactic categories) and to compare them with meaning resolution processes in the absence of syntacti- 
cally biasing information (i.e., for lexical ambiguity within a syntactic category).

In a number of studies, the processing of lexically ambiguous words whose meanings share a syntactic category has been examined. Across different tasks, two factors consistently have been found to influence the resolution process: context and meaning dominance (see, e.g., Duffy et al., 1988; Folk \& Morris, 1995; Rayner et al., 1994; Seidenberg et al., 1982; Simpson \& Burgess, 1985; Simpson \& Foster, 1986; Simpson \& Kellas, 1988; Tabossi, 1988). Meaning dominance refers to the relationship between the meanings of an ambiguous word, with balanced words having relatively equally likely meanings and biased words having one dominant interpretation and one less likely subordinate interpretation. In a series of eye movement studies (e.g., Binder \& Morris, 1995; Dopkins et al., 1992; Duffy et al., 1988; Folk \& Morris, 1995; Rayner \& Duffy, 1986; Rayner \& Frazier, 1989; Rayner et al., 1994; Sereno et al., 1992), the following general pattern of meaning dominance and contextual influences on lexical ambiguity resolution has been found.

When prior context is neutral as to the intended interpretation of the ambiguous word, readers fixate longer on a balanced ambiguous word than on a biased ambiguous word or on an unambiguous control word. Presumably, the two meanings of a balanced ambiguous word are available for selection close in time on the basis of meaning frequency information (Duffy et al., 1988). These meanings then compete for selection, resulting in longer initial reading times relative to an unambiguous control word. The initial assignment of meaning will have to be revised in those cases in which the initially selected meaning is incompatible with subsequent biasing context. In contrast, fixation times on biased ambiguous words do not differ from those on control words. In the absence of prior biasing context, the dominant meaning is available first (Simpson \& Burgess, 1985; Simpson \& Foster, 1986) with little competition for selection from the subordinate meaning.

Conversely, when prior context is semantically consistent with the less likely meaning of an ambiguous word, readers fixate longer on a biased ambiguous word than on a balanced ambiguous word or an unambiguous control word. The inflated processing time on the biased ambiguous word in the presence of prior context that is semantically consistent with the less likely subordinate interpretation has been termed the subordinatebias effect (Rayner et al., 1994). This effect has been interpreted as indicating that context acts to increase the activation of the subordinate interpretation of a biased ambiguous word, making that interpretation available close in time to the dominant interpretation, creating competition. This pattern from eye movement studies is consistent with findings from other tasks that have demonstrated that context can influence the availability of the meanings of semantically ambiguous words (e.g., Seidenberg et al., 1982; Simpson \& Kellas, 1988; Tabossi, 1988).

The subordinate bias effect has proven to be quite robust. Attempts to eliminate the effect by familiarizing read- ers with the subordinate sense, using extended text in which the global discourse is consistent with the subordinate sense, or by repeating the ambiguous item used in its subordinate sense, have all been unsuccessful (see, e.g., Morris \& Binder, 2001; Rayner et al., 1994). This research suggests that the subordinate sense is rarely, if ever, selectively accessed for lexically ambiguous words whose meanings share the same syntactic category in natural text (but see Vu, Kellas, \& Paul, 1998, for an opposing view).

In contrast with the large number of investigationsinto how lexical ambiguity within a syntactic category is processed, much less work has been done in examining the processing of lexical ambiguity that crosses syntactic categories. Furthermore, the purpose of much of the prior work has been to investigate structural ambiguity resolution (e.g., Boland, 1997; Frazier \& Rayner, 1987; MacDonald, 1993). Only a few studies have examined meaning resolution for both semantic- and syntactic-categoryambiguous words in the absence of syntactic ambiguity, which is the focus of the present study.

In a series of studies, Tanenhaus and his colleagues (Seidenberg et al., 1982; Tanenhaus \& DonnenwerthNolan, 1984; Tanenhaus, Leiman, \& Seidenberg, 1979) investigated the availability of the meanings of syntacticcategory-ambiguous (SCA) words in the presence of prior biasing context. For example, Tanenhaus and DonnenwerthNolan (1984) had subjects perform a cross-modal lexical decision task in which subjects listened to sentences ending in a noun-verb (NV) ambiguous word (e.g., prune) and then made a lexical decision to a word related to either the noun or the verb meaning of the ambiguous word. Only one meaning was supported by the context, and the context provided definitive syntacticinformation as to whether the noun or the verb meaning was intended. The control condition involved replacing the ambiguous word in each sentence with an unambiguous control word that was not related to the target word. Tanenhaus and DonnenwerthNolan reported evidence that both meanings of an NV ambiguous word were initially active, regardless of contextual bias. By $200 \mathrm{msec}$, only the context-appropriate interpretation was available, as is the case with the findings from the noun-noun (NN) ambiguity literature (e.g., Swinney, 1979). However, the task did not allow for an examination of meaning resolution processes of the NV ambiguous words - that is, while both meanings were initially active, did they compete for selection, or did the syntactic-category information mediate semantic resolution, eliminating competition?

In a recent study, Seely, Morris, and Schmauder (2002) examined the processing of $\mathrm{NV}$ ambiguous words in structurally ambiguous sentences. They compared reading times on balanced NV ambiguous words with those on unambiguous controls and found ambiguity effects on the ambiguous words under those conditions. These data indicate that, in the absence of prior semantic or syntactic disambiguation, readers must resolve the ambiguity associated with having multiple meanings active, even when those meanings belong to different syntactic categories. 
Related work in the neuropsychological literature suggests that there is a separation of lexical and syntactic processing (Ainsworth-Darnell, Shulman, \& Boland, 1998; Besche et al., 1997; Neville, Nicol, Barss, Forster, \& Garrett, 1991; Osterhout \& Holcomb, 1992). For example, Osterhout and Holcomb presented ERP data that revealed different processing patterns for syntactic anomalies and semantic anomalies. They suggested that these data indicate that syntactic and semantic anomalies are associated with distinct cognitive processes. In keeping with such an interpretation, Besche et al. found evidence of abnormal semantic processing with the preservation of syntactic processing in schizophrenic patients with thought disorders (see also Breedin \& Saffran, 1999). Thus, data has begun to emerge from the neuropsychological literature that syntactic processing and semantic processing are distinct processes (see, also, Hodges, Patterson, \& Tyler, 1994; Ostrin \& Tyler, 1995; and see Martin, 2001, for a review).

The goal of the present studies was to explore meaning resolution for lexically ambiguous words when a word's meanings fall into different syntactic categories. Prior research indicates that both meaning dominance and semantic context influence ambiguity resolution when that ambiguity is within a single syntactic category (for a review, see Rayner \& Morris, 1991). The cross-modal priming studies of Tanenhaus and his colleagues suggest that multiple meanings of a SCA word are initially active (Seidenberg et al., 1982; Tanenhaus \& Donnenwerth-Nolan, 1984; Tanenhaus et al., 1979), as has been found for ambiguous words whose meanings share a single syntactic category. However, the cross-modal naming and lexical decision tasks used in that work do not indicate how the meaning resolution process proceeds when the multiple meanings cross syntactic categories. Of particular interest in the present studies is the role of syntactic information in the semantic-resolution process.

\section{EXPERIMENT 1}

We examined the influence of syntactic-category information on semantic resolution by comparing the processing of lexically ambiguous words whose meanings share a single syntactic category (always the noun category) with that of lexically ambiguous words whose semantic ambiguity crosses syntactic categories (one meaning is in the noun category and one is in the verb category). Participants' eye movements were monitored as they read sentences that contained biased lexically ambiguous words whose meanings were both nouns (e.g., calf), biased lexically ambiguous words whose meanings belonged to two different syntactic categories (e.g., duck), or unambiguous control words. In this task, readers have to analyze sentence structure and resolve the semantic ambiguity in order to comprehend the sentences successfully. This allowed for the examination of the interaction between syntactic and semantic processing during on-line reading. Since we were interested in what effect syntactic-category assignment would have on semantic resolution, our materials were designed in such a way that syntactic information presented prior to the target word unambiguously specified the context-appropriate syntactic-category assignment for the target word. Prior context was structurally unambiguous and semantically consistent with one interpretation of the ambiguous word. In half the cases, this was the subordinate interpretation, and in half the cases it was the dominant interpretation.

We expected to replicate the subordinate bias effect for the NN ambiguous words (see, e.g., Duffy et al., 1988; Rayner et al., 1994). That is, the readers were expected to spend more initial reading time on the ambiguous words with two noun meanings than on unambiguous controls when prior context was consistent with the subordinate interpretation. Given that in prior cross-modal priming studies, evidence has been reported of exhaustive access of the semantic interpretations of SCA words regardless of contextual bias (Seidenberg et al., 1982; Tanenhaus et al., 1979), one might expect to find the subordinate bias effect for the NV ambiguous words as well. According to this account, both meanings would be initially active, regardless of context. Our sentences were designed in such a way that in one condition prior syntactic and semantic context clearly specified that the less likely noun interpretation was intended, setting up the conditions for the subordinate bias effect. However, work by several researchers (Boland, 1993; Gorrell, 1989; O'Seaghdha, 1989, 1997) suggests that syntactic processing could influence semantic resolution. According to such an account, the outcome of syntactic processing could mediate semantic activation in such a way that only the noun interpretation is considered for selection and integration, and, thus, processing difficulty is eliminated.

We also examined how syntactic context influences ambiguity resolution when prior context is consistent with the dominant interpretation of an ambiguous word. Previous research with NN ambiguous words has suggested that readers are easily able to assume the dominant interpretation with prior context that is consistent with that interpretation, with little competition from the subordinate interpretation (see Rayner \& Morris, 1991). We investigated how ambiguity resolution for $\mathrm{NV}$ ambiguous words proceeds under these circumstances.

\section{Method}

Participants. Thirty-six members of the University of South Carolina community participated in this experiment in exchange for course credit. All of the participants had normal uncorrected vision and were native speakers of American English.

Materials. Two types of biased lexically ambiguous words were embedded in sentences: semantically ambiguous words whose meanings share the noun category (NN ambiguous; e.g., calf ), and both semantic- and syntactic-category ambiguous words with semantically distinct noun and verb meanings (NV ambiguous; e.g., $d u c k$ ). An unambiguous control word was matched in length (within one character), overall word frequency, and syntactic category with each ambiguous word. The meaning dominance of the NN ambiguous words was determined by a norming task. In this task, 51 undergraduates were given a booklet containing 25 ambiguous words and 35 filler words. The participants were instructed to write the first 
word that came to mind and then to use the original word in a sentence. The sentence responses were used to confirm the interpretation of the associate. Meaning dominance was calculated as the proportion of responses that were consistent with one of the meanings of the ambiguous word. On the basis of these norms, 18 biased NN ambiguous words with a mean bias of .92 (range, .70-.99) were selected for inclusion in the present experiment. For half of the NN ambiguous words, the dominant interpretation was chosen as the target interpretation, and for half the subordinate interpretation was selected. Eighteen biased NV ambiguous words with a mean bias of .84 (range, .71-.98) were also selected. Meaning dominance for these words was taken from the Francis and Kučera (1982) corpus. Half of the words were verb dominant and half were noun dominant. The noun interpretation was always selected as the target interpretation; for half of the words, it was the dominant interpretation, and for half it was the subordinate. If an ambiguous word had more than two meanings, that word was included in the experiment only if the additional meanings were clearly subordinate. If the additional meanings violated the grammatical category condition for which the word was selected, they were ignored only if they were also semantically related to one of the two primary meanings.

Word frequency was determined according to the Francis and Kučera (1982) corpus. Mean word frequencies, with ranges reported parenthetically, were as follows: $\mathrm{NN}$ ambiguous words with context biased to the dominant meaning, 78 (5-464), and controls, 78 (3467); NN ambiguous words biased to the subordinate meaning, 76 (6-527), and controls, 81 (7-548); NV ambiguous words biased to the dominant noun meaning, 62 (10-235), and controls, 62 (13203); NV words biased to the subordinate noun meaning, 56 (6204), and controls, 58 (6-182).

Sentence frames were constructed for each lexically ambiguous word in such a way that both the ambiguous word and the control word fit smoothly into the sentence frame. Two sentence frames were created for each ambiguous word and its control so that participants could see all of the ambiguous words and their corresponding control words, but in different sentence frames (see Appendix A). The prior context was semantically consistent with one meaning of the ambiguous word (always a noun). Half of the ambiguous words were embedded in sentences in which prior context was semantically consistent with the dominant interpretation of the ambiguous word, and half were embedded in sentences in which the prior context was semantically consistent with its subordinate interpretation. All sentence frames were syntactically unambiguous, allowing only a noun interpretation of the target. For half of the NV ambiguous words, the noun meaning was the dominant interpretation, and for half the noun meaning was the subordinate interpretation. A norming task was conducted to ensure that ambiguous words and control words fit equally well in each sentence frame. Forty undergraduates rated each of the sentences with either the ambiguous or the control word in the target location, and indicated on a 5-point scale how well the target word fit in the sentence. A rating of 5 indicated a very good fit, and a rating of 1 indicated that the word did not fit in the context. The mean rating for NV ambiguous targets disambiguated to the subordinate interpretation was 3.9, and the rating for corresponding controls was 3.7. The mean for $\mathrm{NN}$ ambiguous targets disambiguated to the subordinate interpretation was 3.8 , and that for controls was 3.9. The means for the conditions disambiguated to the dominant interpretation were 4.2 for $\mathrm{NV}$ ambiguous words and 4.1 for the controls; the mean was 4.1 for the $\mathrm{NN}$ ambiguous and 4 for the controls. Standard deviations did not exceed .5 in any single condition. The sentences were also normed to ensure that the participants were successfully arriving at context-appropriate interpretations of the sentence. A separate group of 40 undergraduates saw each sentence with the target word (ambiguous word or control) highlighted and were asked to indicate the meaning of the word in the sentence by selecting from a set of three options. In the case of ambiguous words, two possible meanings of each word were included in the choices. All items were correctly interpreted by more than $90 \%$ of the participants. The mean for every condition reached or exceeded $98 \%$ correct responses.

Procedure. At the beginning of a session, the experimenter prepared a bite bar for each participant to eliminate head movements during testing. The experimenter instructed each participant to read normally for comprehension and informed him or her that "yes or no" comprehension questions would be asked periodically throughout testing. The eye-tracking system was aligned and calibrated for each participant, which took approximately $5 \mathrm{~min}$.

A trial began with a row of five target boxes displayed on the computer screen. The leftmost target box marked the position at which the first letter of a sentence would appear. The participant was told to look at the left box when he or she was ready for a trial to begin, and the experimenter would then present a sentence. When the participant pressed a button to indicate that he or she had finished reading the sentence, it was replaced by the row of target boxes. The experimenter asked a comprehension question on approximately $25 \%$ of the trials. All the participants performed at $90 \%$ accuracy or better on the comprehension questions.

Each participant was tested individually. The participants read six practice sentences to become familiar with the testing procedure prior to reading the experimental sentences. After the practice trials, each participant read 108 sentences. There were 72 experimental sentences, 36 containing an ambiguous word and 36 containing an unambiguous control word. There were an additional 36 filler sentences. Each session lasted approximately $20 \mathrm{~min}$.

The order of presentation of the sentences was randomized for each participant. Eight conditions were formed by the crossing of three factors: ambiguity grammatical category (NN vs. NV), ambiguity (ambiguous word vs. unambiguous control word), and context (consistent with the subordinate vs. with the dominant meaning). All the participants saw all of the conditions with sentence-frame and target-word presentation counterbalanced across participants using a Latin square design.

Apparatus. The participants' eye movements were recorded by a Fourward Technology Dual Purkinje Image eye movement monitoring system. Viewing was binocular, and eye movements were recorded from the right eye. An IBM PS/2 model 80 computer controlled the stimulus display and data storage, and was interfaced with the eye tracker. Each sentence was presented on a single line, with up to 71 characters per line. All of the characters were presented in lowercase, except for the first word of each sentence and proper names. Four character spaces equaled $1^{\circ}$ of visual angle. The eye tracker sampled a participant's eye position every millisecond with a resolution of $10^{\prime}$ of arc. The sentences were displayed on an IBM PS/2 8512 color monitor.

\section{Results and Discussion}

Gaze durations were collected in the target-word region (i.e., the ambiguous word or its unambiguous control word) as a measure of initial processing. Gaze duration is the sum of all initial fixations on a word, including the first fixation and any other fixations until a reader first leaves a word. Rereading was not included in gaze duration. Additionally, two text-integration measures were collected. Spillover is the fixation time on the fixation immediately following the target word and is believed in part to reflect text-integration processes (Rayner \& Duffy, 1986). Second pass time on the target word includes only rereading time on the target.

Standard cutoffs were used to trim the data in such a way that any fixations longer than 1,000 msec or shorter than $120 \mathrm{msec}$ were eliminated from the data. Fixations as 
Table 1

Mean Gaze Duration (in Milliseconds) on the Ambiguous and Control Words When Prior Context was Consistent with the Subordinate Interpretation in Experiment 1

\begin{tabular}{lcc}
\hline & \multicolumn{2}{c}{ Ambiguity } \\
\cline { 2 - 3 } Ambiguity & Noun-Noun & Noun-Verb \\
\hline Ambiguous & 316 & 269 \\
Unambiguous & 279 & 276 \\
Ambiguity effect & +37 & -7 \\
\hline
\end{tabular}

long as $1,000 \mathrm{msec}$ typically are a result of a track loss or an eye blink, and fixations shorter than $120 \mathrm{msec}$ are typically not thought to reflect cognitive processing (Morrison, 1984). Approximately $8 \%$ of the data were lost due to cutoffs or track losses. All analyses were performed across both subject $\left(F_{1}\right)$ and item $\left(F_{2}\right)$ variability.

Gaze Duration. A 2 (ambiguity grammatical category: $\mathrm{NN}$ vs. NV) $\times 2$ (ambiguity: ambiguous word vs. unambiguous control word) analysis of variance (ANOVA) was performed for the two types of ambiguous words and their controls when prior context was consistent with the subordinate interpretation (see Table 1). The ANOVA revealed a significant interaction $\left[F_{1}(1,35)=18.13, M S_{\mathrm{e}}=\right.$ $\left.995, p<.001 ; F_{2}(1,34)=17.96, M S_{\mathrm{e}}=668, p<.001\right]$. The readers spent more time on the $\mathrm{NN}$ ambiguous words than on their controls $\left[F_{1}(1,35)=24.51, M S_{\mathrm{e}}=1,025, p<.001\right.$; $\left.F_{2}(1,17)=18.49, M S_{\mathrm{e}}=863, p<.01\right]$, replicating the subordinate bias effect. The readers did not differ in the amount of time that they spent on the NV ambiguous words versus the controls $\left[F_{1}<1 ; F_{2}(1,17)=1.73, M S_{\mathrm{e}}=\right.$ $474, p>.20]$. Furthermore, there was a significant main effect of ambiguity $\left[F_{1}(1,35)=5.89, M S_{\mathrm{e}}=1,370, p<.05\right.$; $\left.F_{2}(1,34)=7.14, M S_{\mathrm{e}}=668, p<.05\right]$, indicating that the readers spent more time overall on the ambiguous words than on their unambiguous controls. The main effect of ambiguity grammatical category was also significant $\left[F_{1}(1,35)=14.30, M S_{\mathrm{e}}=1,580, p<.001 ; F_{2}(1,34)=14.43\right.$, $\left.M S_{\mathrm{e}}=1,143, p<.01\right]$, as the readers had longer gaze duration times on the $\mathrm{NN}$ ambiguous words and their controls than on the NV ambiguous words and their controls. ${ }^{1}$

A similar $2 \times 2$ ANOVA for the two types of ambiguous words and their controls when prior context was consistent with the dominant noun interpretation was performed, and the means are presented in Table 2. This analysis yielded no significant effects $\left(\right.$ all $\left.F_{\mathrm{S}}<1\right)$, except for ambiguity grammatical category $\left[F_{1}(1,35)=2.68\right.$, $\left.M S_{\mathrm{e}}=1,875, p>.11 ; F_{2}(1,34)=1.34, M S_{\mathrm{e}}=1,561, p>.25\right]$.

Overall, the readers spent more time initially on a lexically ambiguous word whose meanings share a single syntactic category when prior semantic context was consistent with the word's subordinate interpretation. This is a replication of the subordinate bias effect, suggesting that the context increased the activation of the subordinate interpretation so that it was available close enough in time to the dominant interpretation to compete for selection (Duffy et al., 1988; Rayner et al., 1994). In contrast, in the presence of prior syntactic information, the subordinate bias effect was not present when the lexical-semantic am- biguity crossed syntactic categories. This pattern suggests that, when the readers encountered syntactic-category information that unambiguously specified that the noun interpretation of an NV ambiguous word was intended, syntactic information mediated lexical-semantic ambiguity resolution.

No significant effects were associated with either type of ambiguous word when the context was consistent with the dominant interpretation. When both context and meaning dominance are consistent with the dominant interpretation, it is selected with little competition from the subordinate interpretation (see Rayner \& Morris, 1991, for a review).

Spillover. A 2 (ambiguity grammatical category: $\mathrm{NN}$ vs. $\mathrm{NV}) \times 2$ (ambiguity: ambiguous word vs. unambiguous control word) ANOVA was performed on the spillover measure when prior context was consistent with the subordinate interpretation. Processing time for the fixation immediately following an ambiguous word was inflated relative to that following a control word $\left[F_{1}(1,35)=15.5\right.$, $M S_{\mathrm{e}}=61, p<.001 ; F_{2}(1,34)=7.80, M S_{\mathrm{e}}=54, p<.01 ; \mathrm{see}$ Table 3]. Spillover times were shorter for the NN ambiguous words and their controls than for the $\mathrm{NV}$ ambiguous words and their controls $\left[F_{1}(1,35)=21.5, M S_{\mathrm{e}}=54, p<\right.$ $\left..001 ; F_{2}(1,34)=6.44, M S_{\mathrm{e}}=118, p<.05\right]$, and there was no interaction $\left(F_{\mathrm{S}}<1\right)$. Thus, whereas overall the fixation times immediately following an ambiguous word were inflated relative to unambiguous control words, this effect was not significantly different for the NN and NV ambiguous words. This pattern for spillover times indicates that the readers may have experienced some difficulty at integration, in that they had to integrate the less frequent subordinate interpretation into the sentence context (see Duffy et al., 1988, for a similar result). Although differences in spillover between NN and NV conditions did not differ statistically, there was a numerical difference suggesting greater spillover in the NV case. According to pure constraint-based accounts, there should be less difficulty in this case, since the context rules out the verb interpretation. This numerical difference is more consistent with a structure-first account in that it may reflect the conflict between contextual constraint to the noun interpretation and bias to build the simplest structure first, which in this case would mean taking the verb interpretation.

A similar $2 \times 2$ ANOVA was performed for the data when the prior context was consistent with the dominant meaning of an ambiguous word (see Table 4). This analy-

Table 2

Mean Gaze Duration (in Milliseconds) on the Ambiguous and Control Words When Prior Context Biased to the Dominant Interpretation in Experiment 1

Ambiguity

Grammatical Category

\begin{tabular}{lcc}
\cline { 2 - 3 } Ambiguity & Noun-Noun & Noun-Verb \\
\hline Ambiguous & 284 & 272 \\
Unambiguous & 280 & 268 \\
Ambiguity effect & +4 & +4 \\
\hline
\end{tabular}


Table 3

Mean Spillover Times (in Milliseconds per Character) and Second Pass Times (in Milliseconds) on the Target Word When Prior Context Biased to the Subordinate Interpretation in Experiment 1

\begin{tabular}{|c|c|c|c|c|}
\hline \multirow[b]{3}{*}{ Ambiguity } & \multicolumn{4}{|c|}{ Ambiguity Grammatical Category } \\
\hline & \multicolumn{2}{|c|}{ Noun-Noun } & \multicolumn{2}{|c|}{ Noun-Verb } \\
\hline & SO & SP & SO & SP \\
\hline Ambiguous & 44.1 & 83 & 50.7 & 86 \\
\hline Unambiguous & 39.9 & 58 & 44.7 & 42 \\
\hline Ambiguity effect & +4.2 & +25 & +6.0 & +44 \\
\hline
\end{tabular}

Note-SO, spillover times; SP, second pass times.

sis yielded only a significant main effect of ambiguity grammatical category $\left[F_{1}(1,35)=69.34, M S_{\mathrm{e}}=37, p<\right.$ $\left..001 ; F_{2}(1,34)=6.82, M S_{\mathrm{e}}=159, p<.05\right]$. More important, no other effects approached significance [interaction, $F_{1}<1 ; F_{2}(1,34)=1.17, M S_{\mathrm{e}}=48, p>.28$; main effect of ambiguity, $\left.F_{\mathrm{S}} \leq 1\right]$. Thus, there is no evidence that the readers experienced any difficulty integrating the dominant meaning of an ambiguous word relative to an unambiguous control word.

Second pass time on the target word. Second pass time on the target word was also examined as a measure of textintegration processes. The $2 \times 2$ ANOVA when prior context was consistent with the subordinate interpretation revealed that the readers spent more time rereading the ambiguous words than their controls $\left[F_{1}(1,35)=7.6\right.$, $M S_{\mathrm{e}}=5,570, p<.01 ; F_{2}(1,34)=8.25, M S_{\mathrm{e}}=1,759, p<$ $.01]$. This is consistent with the integration difficulty reflected in the spillover measure (see Table 3 ). No other effects were significant $\left(F_{\mathrm{S}}<1.2\right)$. Thus, the two measures of text integration indicate that the readers experienced difficulty with both the NN and the NV ambiguous words to a similar degree, most probably reflecting difficulty integrating the less likely subordinate interpretationinto the sentence representation.

A similar analysis performed on the data when the prior context was consistent with the dominant meaning of an ambiguous word revealed no significant effects (see Table 4). Neither the main effect of ambiguity $\left[F_{1}(1,35)=\right.$ $1.68, M S_{\mathrm{e}}=1,522, p>.20 ; F_{2}(1,34)=1.06, M S_{\mathrm{e}}=1,115$, $p>.30$ ] nor the main effect of ambiguity grammatical category was significant $\left[F_{1}(1,35)=1.85, M S_{\mathrm{e}}=2,564, p>\right.$ $\left..18 ; F_{2}(1,34)=3.45, M S_{\mathrm{e}}=1,486, p>.07\right]$. Additionally, the interaction was not significant $\left[F_{1}(1,35)=1.78, M S_{\mathrm{e}}=\right.$ $\left.1,505, p>.19 ; F_{2}(1,34)=1.58, M S_{\mathrm{e}}=1,115, p>.20\right]$. Thus, the second pass times on the target word were consistent with the spillover data, indicating no significant integration difficulties for the dominant interpretation of an ambiguous word relative to an unambiguous control.

\section{EXPERIMENT 2}

Experiment 1 revealed evidence that syntactic processing can influence the semantic resolution of biased ambiguous words. However, prior context that was semantically biasing had to be employed in order to set up the conditions for the $\mathrm{NN}$ subordinate bias effect. Experiment 2 provides an investigation of the effect of syntactic context alone on semantic resolution, in the absence of semantically biasing context. Balanced lexically ambiguous words were embedded in sentences in which prior syntactic context unambiguously supported a noun interpretation, but in which the prior semantic context was neutral as to the intended interpretation of the ambiguous word. Both ambiguous words whose meanings share a single syntactic category (e.g., pitcher) and ambiguous words whose meanings cross syntactic categories (e.g., relish) were included. This also provided the opportunity to replicate the differential effects of syntactic category and meaning ambiguity observed in Experiment 1.

Previous research has consistently found longer initial fixation times on balanced ambiguous words than on unambiguous controls when prior context is semantically neutral (e.g., Binder \& Morris, 1995; Duffy et al., 1988). Presumably, the two meanings of a balanced word are available close in time and compete for selection. We expected to replicate this effect for the NN ambiguous words. Similarly, Seely et al. (2002) found similar effects for balanced NV ambiguous words when the prior context was also syntactically neutral. If, in Experiment 1, when the semantic ambiguity crossed syntactic categories, the lexical-semantic ambiguity effects were eliminated because the outcome of syntactic processing mediated semantic resolution, then the balanced word effect should also be eliminated for the NV ambiguous words. However, in Experiment 1, consistent semantic and syntactic context was available. In this experiment, only disambiguating syntactic context is available prior to the ambiguous word. It is possible that the outcome of syntactic processing alone may not be sufficient to eliminate the lexical-semantic ambiguity resolution effects for the NV ambiguous words in the absence of semantically biasing information.

\section{Method}

Participants. Thirty University of South Carolina undergraduates participated in this study in exchange for course credit. All the participants were native speakers of American English and had normal uncorrected vision. None had participated in Experiment 1.

Materials. Two types of balanced ambiguous words were included in this study: NN ambiguous words (e.g., pitcher) and lexically ambiguous words whose different meanings cross syntactic categories (NV ambiguous; e.g., relish). For the latter, one meaning

Table 4

Mean Spillover Times (in Milliseconds per Character) and Second Pass Times (in Milliseconds) on the Target Word When Prior Context Biased to the Dominant Interpretation in Experiment 1

\begin{tabular}{|c|c|c|c|c|}
\hline \multirow[b]{3}{*}{ Ambiguity } & \multicolumn{4}{|c|}{ Ambiguity Grammatical Category } \\
\hline & \multicolumn{2}{|c|}{ Noun-Noun } & \multicolumn{2}{|c|}{ Noun-Verb } \\
\hline & $\mathrm{SO}$ & SP & $\mathrm{SO}$ & $\mathrm{SP}$ \\
\hline Ambiguous & 40.7 & 60 & 50.1 & 40 \\
\hline Unambiguous & 40.6 & 43 & 48.0 & 41 \\
\hline Ambiguity effect & +0.1 & +17 & +2.1 & -1 \\
\hline
\end{tabular}

Note-SO, spillover times; SP, second pass times. 
Table 5

Mean Gaze Duration (in Milliseconds) on the Ambiguous and Control Words With Prior Neutral Context in Experiment 2

\begin{tabular}{lcc}
\hline & \multicolumn{2}{c}{$\begin{array}{c}\text { Ambiguity } \\
\text { Grammatical Category }\end{array}$} \\
\cline { 2 - 3 } Ambiguity & Noun-Noun & Noun-Verb \\
\hline Ambiguous & 323 & 298 \\
Unambiguous & 295 & 299 \\
Ambiguity effect & +28 & -1 \\
\hline
\end{tabular}

was a noun and the other was a verb. An unambiguous control word was matched in length (within one character), overall word frequency, and syntactic category with each ambiguous word. The ambiguous words and their controls were embedded in sentences in which prior context was semantically neutral as to the intended interpretation of the ambiguous word. However, the prior context contained syntactic information (e.g., the word the) that disambiguated the NV ambiguous words, specifying that the noun interpretation was intended. Two sentence frames were constructed for each ambiguous word in such a way that both the ambiguous word and the control word fit smoothly into the sentence frame. Example materials are included in Appendix B. These sentences were rated on a 5point scale along with the sentences from Experiment 1 for the fit of the ambiguous words and their matched controls in the context. The means were as follows: $\mathrm{NV}$ ambiguous words, 4.3, and controls, 4.2; NN ambiguous words, 4.1, and controls, 4.3. Standard deviations were less than 0.5 in all conditions. These items were also subjected to the meaning-selection task reported in Experiment 1. Each item was correctly interpreted by at least $90 \%$ of the participants. The mean for each condition reached or exceeded $97 \%$. The sentences were also normed for predictability along with the sentences for Experiment 1 . None of the ambiguous or unambiguous control words was predicted by any of the participants.

Meaning dominance norms were collected as reported in Experiment 1 . The mean bias of the semantically ambiguous words was .63 (range, .50-.68). As in Experiment 1, the meaning dominance for the NV ambiguous words was taken from the Francis and Kučera (1982) corpus. The mean bias of the NV ambiguous words was .63 (range, .50-.68). All other selection criteria were the same as in Experiment 1 . Mean word frequency for the $\mathrm{NN}$ ambiguous words was 37 (3-100), and 35 for controls (6-119); for NV ambiguous words it was 30 (2-172), and 34 for controls (1-183).

The sentences were presented in a different random order to each participant. Four conditions were formed by crossing the two factors of ambiguity grammatical category ( $\mathrm{NN}$ vs. NV) and ambiguity (ambiguous word vs. unambiguous control word). Each participant read a total of 134 sentences: 44 experimental sentences and 90 fillers. The participants saw all of the ambiguous words and their controls, but saw them in different sentence frames.

Procedure. The same procedure was used as in Experiment 1. All the participants performed at or above $90 \%$ accuracy on the comprehension questions.

Apparatus. The same apparatus was used as in Experiment 1.

\section{Results and Discussion}

Reading times were examined in two regions of the sentences: a target-word region consisting of the ambiguous word or its unambiguous control, and a disambiguating region consisting of semantically biasing context that followed the target word. Reading times were examined in the disambiguating region, because that region followed the target word, allowing for an examination of which meaning the readers initially selected in the presence of neutral context (NN words) or only syntactic context ( $\mathrm{NV}$ words). Gaze duration and spillover were calculated in the same manner as in Experiment 1. In addition, quasi-first pass time was calculated in the disambiguating region. This measure was calculated as the sum of all fixations, beginning with the first fixation in the disambiguating region and ending when the reader first left the region or pressed the button to end the trial. This sum does not include any regressions from the region. The sum was divided by the number of characters in the region (milliseconds per character). The same cutoffs were used as in Experiment 1. Approximately $6 \%$ of the data were lost as a result of track losses or short fixations. All analyses were performed across both subject $\left(F_{1}\right)$ and item $\left(F_{2}\right)$ variability.

Gaze duration on the target word. An examination of the means presented in Table 5 reveals that, whereas gaze durations were longer on balanced ambiguous words whose meanings share a single syntactic category than on their unambiguous controls, this effect was not present when the ambiguity crossed syntactic categories. The 2 (grammatical category: $\mathrm{NN}$ vs. $\mathrm{NV}$ ) $\times 2$ (ambiguity: ambiguous word vs. unambiguous control word) ANOVA yielded a reliable interaction in the subjects analysis $\left[F_{1}(1,29)=\right.$ $5.89, M S_{\mathrm{e}}=1,064, p<.05 ; F_{2}(1,42)=3.80, M S_{\mathrm{e}}=1,217$, $p>$.05]. The planned comparisons revealed, as predicted, that the readers spent more time on the balanced NN ambiguous words than on their controls $\left[F_{1}(1,29)=19.85\right.$, $\left.M S_{\mathrm{e}}=598, p<.001 ; F_{2}(1,21)=7.71, M S_{\mathrm{e}}=1,150, p<.05\right]$. This replicates previous findings in the lexical ambiguity literature for balanced words (Duffy et al., 1988) and suggests that the two equally likely meanings of a balanced ambiguous word are available close in time, in the absence of prior biasing context. Interestingly, the NV ambiguous words did not differ from their controls $\left(F_{\mathrm{S}}<1\right)$. Thus, the presence of prior syntactic-category information alone was sufficient to eliminate any potential lexical-semantic ambiguity effects when that ambiguity crossed syntactic categories. This pattern for the balanced words is consistent with that found for biased ambiguous words in Experiment 1 . The basic lexical ambiguity effects are replicated when lexical-semantic ambiguity is within a single syntactic category, but the effects are absent when that ambiguity crosses syntactic categories and prior syntactic context unambiguously indicates that only the noun interpretation is appropriate.

Furthermore, there was a main effect such that the readers spent more time on ambiguous words than on unambiguous controls by subjects $\left[F_{1}(1,29)=6.94, M S_{\mathrm{e}}=807\right.$, $\left.p<.05 ; F_{2}(1,42)=3.48, M S_{\mathrm{e}}=1,217, p=.07\right]$. The main

Table 6

Mean Spillover Times (in Milliseconds per Character) and Mean Quasi-First Pass Time in the Disambiguating Region (in Milliseconds per Character) in Experiment 2

\begin{tabular}{|c|c|c|c|c|}
\hline \multirow[b]{3}{*}{ Ambiguity } & \multicolumn{4}{|c|}{ Ambiguity Grammatical Category } \\
\hline & \multicolumn{2}{|c|}{ Noun-Noun } & \multicolumn{2}{|c|}{ Noun-Verb } \\
\hline & $\mathrm{SO}$ & FP & $\mathrm{SO}$ & FP \\
\hline Ambiguous & 49.0 & 44.0 & 47.8 & 40.5 \\
\hline Unambiguous & 47.6 & 40.4 & 45.4 & 38.9 \\
\hline Ambiguity effect & +1.4 & +3.6 & +2.4 & +1.6 \\
\hline
\end{tabular}

Note-SO, spillover times; FP, quasi-first pass times. 
effect of ambiguity grammatical category (NN vs. NV) was not significant $\left[F_{1}(1,29)=1.66, M S_{\mathrm{e}}=1,861, p>.20\right.$; $\left.F_{2}(1,42)=1.42, M S_{\mathrm{e}}=1,769, p>.24\right]$.

Spillover. The means for the spillover measure are presented in Table 6 . The 2 (ambiguity grammatical category: $\mathrm{NN}$ vs. $\mathrm{NV}) \times 2$ (ambiguity: ambiguous word vs. unambiguous control word) ANOVA performed on the duration of the first fixation following the target word revealed only a main effect of ambiguity by subjects $\left[F_{1}(1,29)=\right.$ $\left.4.80, M S_{\mathrm{e}}=23, p<.05\right]$, but not by items $\left[F_{2}(1,42)=1.85\right.$, $\left.M S_{\mathrm{e}}=44, p>.18\right]$. Neither the main effect of grammatical category $\left[F_{1}(1,29)=2.33, M S_{\mathrm{e}}=37, p>.13 ; F_{2}<1\right]$ nor the interaction was significant $\left(F_{\mathrm{S}}<1\right)$. The negligible spillover effects suggest that the readers' initial difficulty on the NN balanced words was largely resolved on the target word itself.

Quasi-First Pass Time in the Disambiguating Region. We also examined processing time in the disambiguating region in Experiment 2, since that region followed the ambiguous target word. An examination of the means in Table 6 indicates that the readers spent more time in the disambiguating region following the $\mathrm{NN}$ ambiguous words than following their unambiguous controls, as confirmed by a planned comparison $\left[F_{1}(1,29)=4.86, M S_{\mathrm{e}}=\right.$ $\left.40.1, p<.05 ; F_{2}(1,21)=5.63, M S_{\mathrm{e}}=21.9, p<.05\right]$. However, reading time in the disambiguating region did not differ for the NV ambiguous words and their controls $\left[F_{1}(1,29)=1.04, M S_{\mathrm{e}}=38.7, p>.30 ; F_{2}(1,21)=1.06, M S_{\mathrm{e}}=\right.$ $26.6, p>.30]$.

The 2 (grammatical category: NN vs. NV) $\times 2$ (ambiguity: ambiguous word vs. unambiguous control word) ANOVA revealed a significant main effect of ambiguity $\left[F_{1}(1,29)=7.78, M S_{\mathrm{e}}=26.4, p<.01 ; F_{2}(1,42)=5.56\right.$, $\left.M S_{\mathrm{e}}=24.2, p<.05\right]$. The main effect of grammatical category was significant by subjects $\left[F_{1}(1,29)=14.50\right.$, $\left.M S_{\mathrm{e}}=12.9, p<.01\right]$ but not by items $\left[F_{2}(1,42)=1.7\right.$, $\left.M S_{\mathrm{e}}=112, p>.19\right]$. This effect reflected the fact that gaze duration times were longer for the disambiguating region when it followed the $\mathrm{NN}$ ambiguous words or their controls (42.2 msec per character) than when it followed the $\mathrm{NV}$ ambiguous words or their controls $(39.7 \mathrm{msec}$ per character). The interaction was not significant $\left(F_{\mathrm{S}}<1\right)$.

The finding of increased processing time in the disambiguating region following $\mathrm{NN}$ ambiguous words in comparison with unambiguous controls replicates the findings of Duffy et al. (1988). The increased processing time suggests that on some trials the readers initially selected an interpretation of the $\mathrm{NN}$ ambiguous word that turned out to be inconsistent with the subsequent disambiguating region. The fact that there was no evidence of increased processing time in the disambiguating region following the $\mathrm{NV}$ words is evidence that the readers initially selected the contextually appropriate interpretation.

\section{GENERAL DISCUSSION}

In two experiments, the presence of unambiguous structural context prior to a lexically ambiguous word eliminated meaning resolution effects when the ambiguity crossed syntactic categories. In Experiment 1, the context was semantically and syntactically consistent with the subordinate interpretation of the biased ambiguous word, and the standard lexical-semantic ambiguity effect was eliminated. In Experiment 2, prior context was semantically neutral. Only the structural information presented prior to a balanced ambiguous word indicated which meaning of the ambiguous word was intended. In this case, the syntacticcategory information alone was sufficient to eliminate lexical ambiguity effects during on-line reading. Thus, the data from the two experiments indicate that syntacticcategory assignment can mediate meaning resolution.

Previous attempts to eliminate the subordinate bias effect for biased ambiguous words whose meanings share a single syntactic category have been unsuccessful, even with prior context that was strongly biased toward the subordinate interpretation (see, e.g., Binder \& Rayner, 1998; Morris \& Binder, 2001). Although Vu et al. (1998) reported the elimination of the subordinate bias effect with strong prior context, these results have been subject to alternative interpretations (Binder \& Rayner, 1998; Wiley \& Rayner, 2000). In the Vu et al. study, participants responded to a related probe word that immediately followed a sentence ending with an ambiguous word. A recent paper by Peleg, Giora, and Fein (2001) reported a replication of $\mathrm{Vu}$ et al.'s results even in the absence of the ambiguous word, suggesting that the effects observed by $\mathrm{Vu}$ et al. were the product of the relationship between sentence context and probe as opposed to that of the relationship of the context to the ambiguous word. In summary, previous attempts to eliminate the subordinate bias effect via contextual manipulations have been at best equivocal. Although we did not directly compare the processing of $\mathrm{NV}$ ambiguous words in syntactically ambiguous versus unambiguous contexts, the data from Experiment 1 clearly distinguish resolution of lexical ambiguity within a syntactic category from lexical ambiguity resolution across syntactic-category boundaries by demonstrating the absence of standard ambiguity effects in the cases involving SCA words.

In both experiments, we found that prior syntactic context is sufficient to override any competition from the contextually inappropriate interpretation when lexicalsemantic ambiguity crosses syntactic categories. One interpretation of these findings is that the syntactically appropriate interpretation is selectively accessed. However, evidence from cross-modal naming and lexical decision suggests that multiple meanings of SCA words are initially active regardless of prior semantic or syntactic contextual bias (Seidenberg et al., 1982; Tanenhaus \& DonnenwerthNolan, 1984; Tanenhaus et al., 1979). How do we reconcile these findings? One explanation of the present findings that is consistent with the cross-modal priming studies is that both meanings of the SCA words are initially lexically active, regardless of contextual bias. However, because the prior syntactic context unambiguously indicates that a noun is intended, the results of syntactic pro- 
cessing support only the noun meaning. That interpretation is available first for selection, on the basis of the outcome of syntactic processing, even when the verb interpretation is the dominant interpretation, as it was in Experiment 1, or when both meanings are equally likely on the basis of meaning dominance information, as in Experiment 2 . That is, syntactic context reorders the availability of the meanings so that, although both meanings are initially lexically active, there is no competition between them, because only the syntactically appropriate noun interpretation is considered for selection and integration. Thus, the outcome of syntactic processing constrains semantic resolution, but does not necessarily result in selective access. The spillover and second pass times on the target word in Experiment 1 are consistent with this interpretation. Both of these measures revealed increased processing time in the ambiguous case, regardless of ambiguity type (within or across syntactic category). If access were selective, there should be no effect of ambiguity in these measures.

These results could be accounted for with a constraintsatisfaction model (see, e.g., MacDonald et al., 1994; Tabor \& Tanenhaus, 1999; Trueswell \& Tanenhaus, 1994). According to a constraint-based interactive-activation account, ambiguity resolution is a constraint-satisfaction problem in which all available information is used to constrain the resolution process. From this viewpoint, in Experiment 1 semantic and syntactic contextual information converge to yield the noun interpretation of the ambiguous word, despite the fact that meaning dominance information favored the dominant verb interpretation. The syntactic contextunequivocallysupports the noun interpretation and excludes the verb interpretation, and, as such, it is more constraining than the meaning bias inherent in the word. Given that the syntactic context rules out the alternative (verb) interpretation, it is also more constraining than is semantic contextual bias for NN ambiguous words. Semantic context provides bias in favor of one interpretation but does not exclude the other interpretation. Because the syntactic context is so constraining, and both semantic and syntactic information converge on a single interpretation, the system settles on a single interpretation quickly and easily. Thus, there is no evidence of processing difficulty on the part of the reader. In Experiment 2, the same thing occurs even in the absence of semantic contextual constraints. Syntactic information alone provides sufficient constraint so that no ambiguity effect is observed.

An alternative interpretation of the findings reported here is that in order for the semantic-resolution process to be completed, a rudimentary syntactic analysis must first occur (see, e.g., O'Seaghdha, 1997)—that is, syntactic processing precedes semantic processing. According to this account, the ambiguous word is assigned to the syntactic category "noun" independently of analysis of word meaning. There is only one meaning associated with the noun interpretation; thus, there is no meaning ambiguity to resolve and no evidence of processing difficulty. The results of syntactic processing were available early enough to eliminate any semantic-resolution difficulties that would result from having multiple meanings associated with a single spelling active close in time. This implies a hierarchical architecture in which syntactic-category assignment is not only distinct from semantic analysis but also precedes it. Our data do not discriminate between the constraint-based and the syntax-first accounts.

It is important to note that the present experiments did not include any structural ambiguity. In situations in which readers also face structural ambiguity, we would expect to see qualitative differences in the pattern of processing for sentences that contained SCA words. In such a case, other types of information that have been suggested as influencing syntactic ambiguity resolution, but that were not relevant to the situation presented in our experiments, might also exert their influence. For example, Frazier and Rayner (1987) and MacDonald (1994) demonstrated that when SCA words are processed under conditions of structural ambiguity, processing difficulties emerge in the posttarget structural-disambiguating region. MacDonald (1994) attributed these differences to probabilistic constraints, whereas Frazier and Rayner attributed similar results to delayed application of structure-building principles. More recent papers have expanded on this debate (e.g., Altmann \& Steedman, 1988; Boland, 1997; Britt, Perfetti, Garrod, \& Rayner, 1992; Spivey-Knowlton \& Tanenhaus, 1994; Trueswell, Tanenhaus, \& Garnsey, 1994). Our data do not speak to this issue. Rather, our data demonstrate that in the absence of syntactic ambiguity, syntactic-category information mediates the semanticresolution process.

\section{REFERENCES}

Ainsworth-Darnell, K., Shulman, H. G., \& Boland, J. E. (1998). Dissociating brain responses to syntactic and semantic anomalies: Evidence from event-related potentials. Journal of Memory \& Language, 38, 112-130.

Altmann, G. T. M., \& Steedman, N. (1988). Interaction with context during human sentence processing. Cognition, 30, 191-238.

Besche, C., Passerieux, C., Hardy-Bay le, M. C., Sarfati, Y., Segui, J., \& LAURENT, J. P. (1997). Syntactic and semantic processing in schizophrenic patients evaluated by lexical-decision tasks. Neuropsychology, 11, 498-505.

BINDER, K. S., \& Morris, R. K. (1995). Eye movements and lexical ambiguity resolution: Effects of prior encounter and discourse topic. Journal of Experimental Psychology: Learning, Memory, \& Cognition, 21, 1186-1196.

BindER, K. S., \& RAYNer, K. (1998). Contextual strength does not modulate the subordinate bias effect: Evidence from eye fixations and selfpaced reading. Psychonomic Bulletin \& Review, 5, 271-276.

Boland, J. E. (1993). The role of verb argument structure in sentence processing: Distinguishing between syntactic and semantic effects. Journal of Psycholinguistic Research, 22, 133-152.

BoLAND, J. E. (1997). Resolving syntactic category ambiguities in discourse context: Probabilistic and discourse constraints. Journal of Memory \& Language, 36, 588-615.

BREEdin, S., \& SAFFran, E. (1999). Sentence processing in the face of semantic loss: A case study. Journal of Experimental Psychology: General, 128, 547-562.

Britt, M. A., Perfetti, C. A., Garrod, A., \& Rayner, K. (1992). Parsing in discourse: Context effects and their limits. Journal of Memory \& Language, 31, 293-314.

Dopkins, S., Morris, R. K., \& RAYNeR, K. (1992). Lexical ambiguity 
and eye fixations in reading: A test of competing models of lexical ambiguity resolution. Journal of Memory \& Language, 31, 461-476.

DufFy, S. A, Morris, R. K., \& RAYNer, K. (1988).Lexical ambiguity and fixation times in reading. Journal of Memory \& Language, 27, 429-446.

FolK, J. R., \& Morris, R. K. (1995). The use of multiple lexical codes in reading: Evidence from eye movements, naming time, and oral reading. Journal of Experimental Psychology: Learning, Memory, \& Cognition, 21, 1412-1429.

FrancIS, W. N., \& KuČERA, H. (1982). Frequency analysis of English usage: Lexicon and grammar. Boston: Houghton Mifflin.

FrAZIER,L., \& RAYNER, K. (1987). Resolution of syntactic category ambiguities: Eye movements in parsing lexically ambiguous sentences. Journal of Memory \& Language, 26, 505-526.

GoRRELL, P. (1989). Establishing the loci of serial and parallel effects in syntactic processing. Journal of Psycholinguistic Research, 18, 61-73.

Hodges, J., Patterson, K., \& Tyler, L. (1994). Loss of semantic memory: Implications for the modularity of mind. Journal of Cognitive Neuropsychology, 11, 505-542.

MacDonald, M. C. (1993). The interaction of lexical and syntactic ambiguity. Journal of Memory \& Language, 32, 692-715.

MacDonald, M. C. (1994). Probabilistic constraints and syntactic ambiguity resolution. Language \& Cognitive Processes, 9, 157-201.

MacDonald, M. C., Pearlmutter, N. J., \& Seidenberg, M. S. (1994). Lexical nature of syntactic ambiguity resolution. Psychological Review, 101, 676-703.

Martin, R. (2001). Sentence comprehension. In B. Rapp (Ed.), The handbook of cognitive neuropsychology: What deficits reveal about the human mind (pp. 349-373). Philadelphia: Psychology Press.

Morris, R. K., \& Binder, K. S. (2001). What happens to the unselected meaning of an ambiguous word in skilled reading? In D. S. Gorfein (Ed.), On the consequences of meaning selection: Perspectives on resolving lexical ambiguity (pp. 139-153). Washington, DC: APA.

Morrison, R. E. (1984). Manipulation of stimulus onset delay in reading: Evidence for parallel programming of saccades. Journal of Experimental Psychology: Human Perception \& Performance, 10, 667-682.

Neville, H., Nicol, J. L., Barss, A., Forster, K. I., \& Garrett, M. F. (1991). Syntactically based sentence processing classes: Evidence from event-related brain potentials. Journal of Cognitive Neuroscience, 3, 155-170.

O'SEAGHDHA, P. G. (1989). The dependence of lexical relatedness effects on syntactic connectedness. Journal of Experimental Psychology: Learning, Memory, \& Cognition, 15, 73-87.

O'Seaghdha, P. G. (1997). Conjoint and dissociable effects of syntactic and semantic context. Journal of Experimental Psychology: Learning, Memory, \& Cognition, 23, 807-828.

Osterhout, L., \& Holcomb, P. J. (1992). Event-related brain potentials elicited by syntactic anomaly. Journal of Memory \& Language, 31, 785-806.

Ostrin, R. \& TYLER, L. (1995). Dissociations of lexical function: Semantics, syntax, and morphology. Journal of Cognitive Neuropsychology, 12, 345-389.

Peleg, O., Giora, R, \& Fein, O. (2001). Salience and context effects: Two are better than one. Metaphor \& Symbol, 16, 173-192.

RAYNER,K., \& DufFy, S. A. (1986). Lexical complexity and fixation times in reading: Effects of word frequency, verb complexity, and lexical ambiguity. Memory \& Cognition, 14, 191-201.

RAYNer, K., \& Frazier,L. (1989). Selection mechanisms in reading lexically ambiguous words. Journal of Experimental Psychology: Learning, Memory, \& Cognition, 15, 779-790.

RAYNER, K., \& MorRIS, R. K. (1991). Comprehension processes in reading ambiguous sentences: Reflections from eye movements. In G. B. Simpson (Ed.), Understanding word and sentence (Advances in Psychology, No. 77, pp. 175-198). Amsterdam: Elsevier, North-Holland.

Rayner, K., Pacht, J. M., \& Duffy, S. A. (1994). Effects of prior en- counter and global discourse bias on the processing of lexically ambiguous words: Evidence from eye fixations. Journal of Memory \& Language, 33, 527-544.

Seely, R. E., Morris, R. K., \& Schmauder, A. R. (2002). Readers' use of syntactic and semantic context in lexical semantic and syntactic category ambiguity resolution. Manuscript submitted for publication.

Seidenberg, M. A., Tanenhaus, M. K., Leiman, J. M., \& BienKOWSKI, M. (1982). Automatic access of the meanings of ambiguous words in context: Some limitations of knowledge-based processing. Cognitive Psychology, 14, 489-537.

Sereno, S. C., Pacht, J. M., \& Rayner, K. (1992). The effect of meaning frequency on processing lexically ambiguous words: Evidence from eye fixations. Psychological Science, 3, 296-300.

Simpson, G. B., \& BurgEss, C. (1985). Activation and selection processes in the recognition of ambiguous words. Journal of Experimental Psychology: Human Perception \& Performance, 11, 28-39.

Simpson, G. B., \& Foster, M. R. (1986). Lexical ambiguity and children's word recognition. Developmental Psychology, 22, 147-154.

Simpson, G. B., \& Kellas, G. (1988). Dynamic contextual processes and lexical access. In D. S. Gorfein (Ed.), Resolving semantic ambiguity (pp. 40-56). New York: Springer-Verlag.

Spivey-Knowlton, M., \& Tanenhaus, M. K. (1994). Referential context and syntactic ambiguity resolution. In C. Clifton, L. Frazier, \& K. Rayner (Eds.), Perspectives on sentence processing (pp. 415-439). Hillsdale, NJ: Erlbaum.

SWINNEY, D. A. (1979). Lexical access during sentence comprehension: $(\mathrm{Re})$ consideration of context effects. Journal of Verbal Learning \& Verbal Behavior, 18, 645-659.

TABor, W., \& Tanenhaus, M. K. (1999). Dynamical models of sentence processing. Cognitive Science, 23, 491-515.

TABossi, P. (1988). Accessing lexical ambiguity in different types of sentential contexts. Journal of Memory \& Language, 27, 324-340.

Tanenhaus, M. K., \& Donnenwerth-Nolan, S. (1984). Syntactic context and lexical access. Quarterly Journal of Experimental Psychology, 36A, 649-661.

Tanenhaus, M. K., Leiman, J. M., \& Seidenberg, M. A. (1979). Evidence for multiple stages in the processing of ambiguous words in syntactic contexts. Journal of Verbal Learning \& Verbal Behavior, 18, 427-440.

Trueswell, J. C., \& Tanenhaus, M. K. (1994). Toward a lexicalist framework for constraint-based syntactic ambiguity. In C. Clifton, L. Frazier, \& K. Rayner (Eds.), Perspectives on sentence processing (pp. 155179). Hillsdale, NJ: Erlbaum.

Trueswell, J. C., Tanenhaus, M. K., \& Garnsey, S. M. (1994). Semantic influences on parsing: Use of thematic role information on syntactic disambiguation. Journal of Memory \& Language, 33, 285-318.

Vu, H., Kellas, G., \& Paul, S. T. (1998). Sources of sentence constraint on lexical ambiguity resolution. Memory \& Cognition, 26, 979-1001.

Wiley, J., \& RAYNeR, K. (2000). Effects of titles on the processing of text and lexically ambiguous words: Evidence from eye movements. Memory \& Cognition, 28, 1011-1021.

\section{NOTE}

1. To ensure that the differences in processing on the NN and NV ambiguous words were not a result of greater predictability in the NV items, 80 participants completed a cloze task. They were given the sentences up to but not including the target words and were asked to complete the sentences. Ambiguous and control targets were given as completions less than $3 \%$ of the time, with two exceptions. The words sock (an NV ambiguous word) and speaker (an NN item) were predicted almost $40 \%$ of the time. Since these words occurred in dominant context conditions, they do not compromise the observed processing differences between the two ambiguity types in the subordinate context conditions. 


\section{APPENDIX A \\ Materials Used in Experiment 1}

The ambiguous word is underlined and the unambiguous control word is in parentheses.

\section{Noun-Noun Ambiguous}

\section{Biased to the Subordinate Interpretation}

1a. Mary went to the fireplace and picked up the poker (bucket) from the ledge.

1b. Cindy hit the burglar over the head with a poker (bucket) and ran for help.

2a. When Todd heard the loud racket (gunfire) upstairs, he reported it.

2 b. Jean cherished the silence after listening to the racket (gunfire) for hours.

3a. After the scandal, the advisor had to resign from the cabinet (academy) in June.

3b. The President appointed the admiral to the cabinet (academy) after the election.

4a. Les threw the ball and hit Ted on the calf (thigh) during the dodge ball game.

4b. While jogging, Cathy pulled a muscle in her calf (thigh) and had to stop.

$5 \mathrm{a}$. The government forced the Indians to live on/in the reservation (wilderness) in Texas.

$5 \mathrm{~b}$. The army told the Sioux that it would protect the reservation (wilderness) forever.

6a. At the convent, Sister Marie prefers to wear a heavy habit (fabric) year round.

6b. The nun searched her closet to find her new habit (fabric) for evening mass.

7a. Before the game, the baseball team went to the diamond (stadium) to practice.

$7 \mathrm{~b}$. The catcher watched as the ball was hit out of the diamond (stadium) last night.

$8 \mathrm{a}$. The waiter spilled the port (beer) in the customer's lap.

8 b. Marcus asked the bartender for a bottle of port (beer) and a cup of water.

9a. The golf pro showed the player a new golf course (system) to use that afternoon.

9b. The NASCAR officials said that the old race course (system) was too dangerous.

\section{Biased to the Dominant Interpretation}

1a. The police officer arrested the crook (felon) who robbed the gas station.

1b. After his release from prison, the old crook (felon) turned his life around.

2a. The carpenter measured the wood with the new ruler (gadget) that he bought.

$2 b$. The artist drew straight lines with the ruler (gadget) that Jim gave to her.

3a. The students went to the assembly and listened to the speaker (speech) today.

3b. Protesters gathered in the street to hear the famous speaker (speech) at noon.

4a. The expensive scarf was made out of an imported yarn (silk) from Europe.

$4 \mathrm{~b}$. The seamstress searched her scrap bag for yarn (silk) to match the hat.

5a. Lisa longed to fly like the cardinal (airplane) that she saw above the trees.

$5 \mathrm{~b}$. The beautiful wings of the cardinal (airplane) glistened in the sun.

6a. George was scared when he heard about the legend (mystery) of the haunted house.

$6 \mathrm{~b}$. Tia loved the local folklore and often recited a legend (mystery) to visitors.

7a. One of Jody's favorite things to eat is fresh corn (peas) from the garden.

7b. Cliff went to the supermarket to buy a can of corn (peas) and some rice.

8a. When Lucy looked in the mirror she saw a reflection (policeman) that startled her. 8 b. Lyn sat by the pond in the park watching a reflection (policeman) as her son slept.

9a. I wanted to join the group but they accepted only one member (woman) this year. $9 \mathrm{~b}$. The delegation needed to elect one member (woman) to present their position.

\section{Noun-Verb Ambiguous}

\section{Biased to the Subordinate Interpretation}

1a. Wendy was in a bad mood and acted like an awful jerk (witch) all afternoon.

1b. When his mom said no, Mark called her a mean jerk (witch) and was sent to bed.

2a. The game warden knew that the injured duck (sheep) was not going to live.

$2 \mathrm{~b}$. Eric went by the pond at the petting zoo to feed the $d u c k$ (sheep) and goose.

3a. As the cat neared the nest, it pounced on a baby swallow (rabbit) and killed it.

3b. By a tree in the woods, Liz saw a bluejay and a swallow (rabbit) that were hurt.

4a. Sam went to the bike shop to get a new spoke (jacket) for the big race.

4b. Biking through Utah, the cyclist lost a spoke (jacket) in the mountains.

5a. Bob had a hard decision to make, so he asked a trusted peer (adult) for help.

$5 b$. Tom hated drugs, but he was pressured by a respected peer (adult) to try them. 
6a. The poacher threw the bloody hide (cloth) into the back of his truck. $6 \mathrm{~b}$. The settler stretched the hide (cloth) over the window to block the wind.

7a. At the music festival, the child danced to the beat (song) and sang out loud.

$7 \mathrm{~b}$. Frank's radio is so loud that I can hear the beat (song) through the walls.

8a. During our trip to the national park, I saw a wild bear (horse) in a field.

$8 \mathrm{~b}$. The most popular act at the circus was the trained horse (bear) from France.

9a. The archery students put the arrows back in the quiver (basket) after class.

9b. The expert marksman took the third arrow from the quiver (basket) and took aim.

\section{Biased to the Dominant Interpretation}

1a. The baker took the bread from the oven and put the loaf (tray) on the shelf.

1 b. Fred was shocked at how moldy the loaf (tray) of cheese on the counter was.

2a. The hunter took the antlers but left the pelt (skull) in the woods.

2b. As a trophy of his first kill, Scott kept an animal pelt (skull) in his den.

3a. Monica made a hand puppet from the old sock (glove) her dad gave to her.

$3 \mathrm{~b}$. Kim got out the sewing kit to mend the hole in her sock (glove) this morning.

4a. The jockey walked the mare into the stall (barn) after their workout.

$4 \mathrm{~b}$. The farm hand had to clean the stall (barn) and put down fresh hay.

5a. Phil wanted to clean his messy room because there was stuff (dirt) everywhere.

5 b. The filthy dog dumped the trashcan, leaving stuff (dirt) all over the floor.

6a. Since my family likes to swim, we vacation at the coast (lake) every year.

6b. After the heavy rains, there was flooding near the coast (lake) this year.

7a. The farmer predicted that the weather this season (summer) would be hot and dry.

7b. After a slow winter, the airline said that this season (summer) would be busy.

8a. The store's grand opening was promoted with a big sign (event) on Main Street. 8 b. Sue advertised at the fair, hoping that the sign (event) would help business.

9a. The bus driver tore my ticket and gave me the stub (token) in return.

$9 \mathrm{~b}$. Renee looked for a bank receipt but only found a stub (token) from the subway.

\section{APPENDIX B}

Materials Used in Experiment 2

The ambiguous word is underlined and the unambiguous control word is in parentheses.

\section{Noun-Noun Ambiguous}

1a. The lady had the organ ( piano) for years but never played it.

1b. Paul put the organ (piano) near the soloist so he could cue her on time.

2a. I noticed that the pupil (nerve) in her left eye was damaged badly.

2 b. Rob said that the pupil (nerve) was diseased and would cause blindness soon.

3a. I heard that the chest (bullet) was locked up in the attic.

$3 \mathrm{~b}$. The man said that the chest (bullet) was buried on a remote island.

4a. My dad took the pipe (cigar) outside so that he could smoke it.

4b. My grandpa put the pipe (cigar) in his mouth and lit it with a match.

5a. Carol knew that the deed (creed) was typed by her secretary this morning.

5 b. Ken knew that the deed (creed) was a legal document so he put it in the safe.

6a. April used the straw (spoon) to stir her glass of iced tea.

6b. Liz said that the straw (spoon) the waiter put in her drink was dirty.

7a. Jesse had the mole (wart) on the end of his nose removed by a doctor.

7b. Christy claimed that the mole (wart) on her face was really a beauty mark.

8 a. Joe must have the cane (wheat) harvested and sold to buy a new tractor.

8 b. I knew that the cane (wheat) would not grow once the farm was flooded.

9a. Eric noticed that the yard (hill) next door needed to be mowed very badly. 9 b. I was told that the yard (hill) by the church was a cemetery years ago. 


\section{APPENDIX B (Continued)}

10a. Steve knew that the panel (jury) could decide to send him to prison for life.

10b. Tony said that the panel (jury) was comprised of citizens from the community.

11a. Ella saw the pitcher (catcher) for her softball team practicing before the game.

11b. Carla had the pitcher (catcher) for the Atlanta Braves give her a signed ball.

\section{Noun-Verb Ambiguous}

1a. Laurie took the prune (grape) out of the fruit bowl and ate it.

1b. Rachel placed the prune (grape) and the banana in the juicing machine.

2a. Don kept the barge (raft) near the shore during the storm.

2b. Lee let the barge (raft) float freely down the river.

3a. Mitch put the dice (token) on the casino table and left.

3b. Whitney said that the dice (token) were/was carved out of ivory from Africa.

4a. Ralph knew that the pants (shirt) were/was two sizes too small for him.

4b. Jeff said that the pants (shirt) were/was made of cotton and polyester.

5a. Lee kept the fawn (puppy) until it was old enough to be on its own.

5b. She watched the fawn (puppy) walk to its mother for food.

6a. Ann put the hamper (basket) in the laundry room by the washing machine.

6b. Carlos said that the hamper (basket) was full of dirty clothes already.

7a. Mark noticed that the count (king) was wearing an expensive sword from Italy.

7b. Jason said that the count (king) was engaged to a princess last year.

8a. Kim took the harp (cello) to the repair shop to be tuned before the concert.

8b. Jen sold the harp (cello) when she quit playing in the orchestra last year.

9a. The boy chose to have the waffle (pancake) for breakfast with some juice.

$9 \mathrm{~b}$. Clint let the waffle (pancake) soak in syrup before he ate it.

10a. Tina put the relish (onion) in the potato salad this morning.

10b. Barb placed the relish (onion) on the hot dog and on the hamburger.

11a. Tia said that the park (river) was a popular place for camping all year long.

$11 \mathrm{~b}$. Bill said that the park (river) was polluted by the nearby factory

(Manuscript received August 8, 2001;

revision accepted for publication September 5, 2002.) 